$2 \begin{aligned} & \text { Pesquisa Florestal Brasileira } \\ & \text { Brazilian Journal of Forestry Research } \\ & \text { www.cnpf.embrapa.br/pfb }\end{aligned}$

\title{
Quintais caseiros em Machipanda, distrito de Manica, Moçambique
}

\author{
Clemencia Carlos Eduardo Chitsondzo', Ivan Crespo Silva² \\ ${ }^{1}$ Instituto Superior Politécnico de Manica, Campos de Matsinho, Distrito de Gondola, Estrada Nacional $\mathrm{n}^{\circ}$ 6, desvio nas antenas km 4,5, Manica, Moçambique \\ ${ }^{2}$ Universidade Federal do Paraná, Av. Pref. Lothário Meissner, 900, Jardim Botânico, Campus III, CEP 80210-170 , Curitiba, Paraná, Brasil
}

\author{
"Autor correspondente: \\ cchitsondzo@yahoo.com.br \\ Termos para indexação: \\ Sistemas agroflorestais \\ Hortas familiares \\ Agricultura de subsistência \\ Agrobiodiversidade \\ Index terms: \\ Agroforestry systems \\ Family gardens \\ Subsistence farming \\ Agrobiodiversity
}

Histórico do artigo:

Recebido em 25/07/2012

Aprovado em 05/06/2013

Publicado em 28/06/2013

doi: 10.4336/2013.pfb.33.74.413.
Resumo - O presente estudo foi feito com o objetivo de caracterizar e avaliar os quintais caseiros em Machipanda, distrito de Manica (Moçambique). Foram realizadas entrevistas com 37 chefes dos agregados familiares. Os resultados obtidos foram analisados com o uso de estatística descritiva. Para facilitar a compreensão e a análise das informações, estas foram agrupadas em uma Matriz SWOT, de maneira que os seus componentes: pontos fortes, pontos fracos, oportunidades e ameaças em relação aos quintais estudados, fossem destacados. Os quintais eram principalmente de forma irregular, áreas variando de 400-5.000 $\mathrm{m}^{2}$ com média de $770,27 \mathrm{~m}^{2}$. Nos quintais caseiros, verificou-se que $90 \%$ praticavam o sistema agrossilvipastoril, enquanto que $10 \%$ praticavam o sistema agrossilvicultural. Nos quintais, foram identificadas 24 espécies arbóreas, pertencentes a 17 famílias botânicas. As famílias mais representativas foram a Rutaceae (4 espécies), Fabaceae (3 espécies), Anacardiaceae, Moraceae e Myrtaceae (2 espécies cada). A agrobiodiversidade existente nos quintais contribui para a segurança alimentar e constitui a principal fonte de renda para o agricultor e sua família. A análise SWOT mostrou que a região é adequada para o desenvolvimento e intensificação dos SAFs e que a prática dos quintais deve ser incentivada na região seguindo preceitos de sustentabilidade socioeconômica e ambiental.

\section{Home garden in Machipanda, Manica district, Mozambique}

\begin{abstract}
This study was carried out in order to characterize and evaluate the home gardens in Machipanda, Manica Province (Mozambique). Interviews were conducted at 37 heads of households. The results were analyzed using descriptive statistics. To facilitate information understanding and analysis, they were grouped into a SWOT Matrix, so that its components: strengths, weaknesses, opportunities and threats in relation to the backyards studied, were highlighted. The backyards were mostly irregular, with an area ranging from $400-5,000 \mathrm{~m}^{2}$ with average of $770.27 \mathrm{~m}^{2}$. In the home gardens it was found that $90 \%$ practiced agrossilvipastoril system and $10 \%$ practiced agroforestry system. Twenty-four tree species belonging to 17 botanic families were identified. The most representative families were Rutaceae ( 4 species), Fabaceae ( 3 species) and Anacardiaceae, Moraceae and Myrtaceae ( 2 species each). The agrobiodiversity present in backyards contribute to food security and constitutes the main source of income for the farmer and his family. The SWOT analysis showed that the region is suitable for development and intensification of agro-forestry systems and that the practice of the backyards should be encouraged in the region under the precepts of socioeconomic and environmental sustainability.
\end{abstract}




\section{Introdução}

Em muitos países em desenvolvimento, o aumento da densidade populacional tem sido cada vez maior e a prática de derrubada e queimada tem contribuído para o empobrecimento dos solos.

Em Moçambique, o aumento da população tem contribuído para uma utilização excessiva dos recursos naturais, devido à exploração destes pelas comunidades para atender as suas necessidades básicas (Macucule, 2005).

Como forma de amenizar os impactos sobre os recursos naturais, vem se desenvolvendo estudos técnicos-científicos sobre alternativas sustentáveis de uso de terra, tais como os sistemas agroflorestais - SAFs (Freitas et al., 2004).

Nos SAFs desenvolvidos na África, o manejo agrícola e florestal é praticado conjuntamente com o uso de diversas atividades culturais. Estes sistemas vêm sendo utilizados há bastante tempo por comunidades rurais e são uma alternativa ao tradicional sistema de derrubada e queimada praticado pelos pequenos agricultores (Fraxe et al., 2007).

Os SAFs são definidos pelo cultivo de espécies arbóreas perenes em combinação interativa com cultivos agrícolas anuais com possível criação de animais, na mesma unidade de terra, de maneira simultânea ou numa sequência temporal (Nair, 1993; Ante, 2006; Van Leeuwen, 2007; Dubois, 2008; César, 2009). Esta prática vem se tornando relevante em áreas agrícolas intensamente exploradas e em locais onde há escassez de espécies florestais (César, 2009).

Apesar de ser uma prática de uso da terra de longa tradição, o interesse por esta prática do ponto de vista científico começou na década de 1970, a partir de dúvidas sobre a eficiência das políticas de desenvolvimento vigentes, que não pareciam se adequar às necessidades e anseios cada vez maiores de pequenos produtores rurais (Nair, 1993).

De acordo com Freitas et al. (2004) existe uma grande variedade de modelos de SAFs, dentre eles destacamse os quintais caseiros, que são muito frequentes nas propriedades rurais.

Os quintais caseiros têm uma longa tradição de uso em países tropicais e consistem em um conjunto de plantas que podem incluir árvores, arbustos, cipós e plantas herbáceas, crescendo no mesmo lugar (Nair, 1993).
A agricultura praticada nos quintais caseiros é baseada no uso de área agrícola reduzida, exigindo do agricultor capacitação para a escolha do modelo de exploração para garantir a sua sustentabilidade ao longo dos anos (Abdo et al., 2008). Quintais caseiros são considerados como sustentáveis ao longo dos anos, pois oferecem uma série de produtos e/ou serviços, diminuindo de forma considerável os gastos da família para obtê-los fora da propriedade (Gazel Filho et al., 2009), e contribuem como complemento alimentar e para a diversificação da dieta das famílias rurais.

O presente trabalho teve como objetivo caracterizar e avaliar os quintais caseiros das comunidades de Machipanda, distrito de Manica, em Moçambique.

\section{Material e métodos}

\section{Descrição da área de estudo}

O distrito de Manica, com uma superfície de $4.594 \mathrm{~km}^{2}$, localiza-se na região central a oeste da província de Manica. .

A localidade de Machipanda localiza-se a $25 \mathrm{~km}$ da sede do distrito de Manica e a $6 \mathrm{~km}$ da fronteira com o Zimbábue. É limitado a norte e a oeste pela República do Zimbábue, a sul pela vila do Posto Administrativo de Machipanda e a leste pela comunidade local.

O clima segundo a classificação climática de Köppen é do tipo temperado úmido $(\mathrm{Cw})$ com precipitação média anual de 1.000. Na região, observam-se duas estações bem distintas, a estação chuvosa e a seca. A estação chuvosa ocorre de novembro a abril e temperatura média anual é de $21,2{ }^{\circ} \mathrm{C}$ (Moçambique, 2005).

O Distrito de Manica é constituído por cadeias montanhosas de Sul a Norte da província, numa faixa fronteiriça com o Zimbábue. Esta formação compreende basaltos, riolitos e lavas alcalinas. A maior parte dos afloramentos formam cristas e cadeias montanhosas que chegam a atingir de 1.500 a $2.000 \mathrm{~m}$ de altitude (Moçambique, 2005).

Da superfície do distrito estimam-se em cerca de 200 mil ha o potencial de terra arável, dos quais apenas 30 mil são explorados pelo setor familiar (Moçambique, 2005).

A agricultura é praticada manualmente em pequenas propriedades familiares, em regime de consorciação de culturas com base em variedades locais. A produção agrícola é predominantemente em condições de 
sequeiro, com riscos altos de perda de colheitas, devido à baixa capacidade de armazenamento e de umidade no solo durante o período de crescimento das culturas (Moçambique, 2005).

Algumas famílias empregam métodos tradicionais de fertilização dos solos, como o pousio das terras, incorporação de resíduos vegetais no solo e uso de estrume ou cinzas (Moçambique, 2005), que, associados ao clima da região, contribuem significativamente para a manutenção da fertilidade dos solos.

\section{Metodologia}

A caracterização e avaliação dos quintais em Machipanda foi feita a partir de levantamentos de campo realizados em novembro de 2008, nas propriedades rurais de agregados familiares (AFs), localizados nos povoados de Mugoriondo, Nhamatanda e Chicueia.

Todo o processo foi decorrente de observação direta e de informações coletadas com a aplicação de entrevista semi-estruturada.

O levantamento foi efetuado em 37 propriedades, representando $10 \%$ da população das três localidades. As unidades familiares foram selecionadas ao acaso,sendo entrevistado cada chefe de agregado familiar.

\section{Levantamento de dados nas propriedades}

As perguntas foram elaboradas paara caracterizar as práticas de agricultura familiar (observação e levantamento de técnicas agrícolas) e formas de integração de componentes agroflorestais nos quintais dos AFs inquiridos.

Foram levantados dados como fonte de renda (principal), espécies, origem e diversidade de espécies existentes no quintal, práticas de manejo e destino da produção.

\section{Análise de dados}

Os dados obtidos nas entrevistas foram organizados e analisados com o uso de estatística descritiva. Os dados foram descritos em tabelas e gráficos para que fosse possível evidenciar as características da distribuição das variáveis avaliadas.

Como forma de desenvolver planos de médio e longo prazos, a partir das informações obtidas pelas entrevistas e com base em percepções próprias, as informações foram agrupadas em uma matriz SWOT: forças (strenghts); fraquezas (weaknesses); oportunidades (opportunities) e ameaças (threats).

A partir da matriz SWOT obteve-se informação qualitativa. Para reduzir a subjetividade da análise
SWOT, fez-se a quantificação das forças e fraquezas usando-se a matriz de avaliação de fatores internos IFE - Intern factor evaluation. Do mesmo modo, fez-se a quantificação das oportunidades e ameaças, usando-se a matriz de avaliação de fatores externos - EFE - Extern factor evaluation.

Para a construção da matriz de avaliação de fatores externos (Tabela 1), inicialmente fez-se a listagem dos fatores externos, começando com as oportunidades, depois as ameaças. Posteriormente, para cada fator externo considerando o grau de importância de cada fator, atribuiu-se pesos que variavam de zero $(0,0)$ a um $(1,0)$, sendo que o somatório total dos pesos atribuídos não deveria ser superior ao valor de um $(1,0)$.

Tabela 1. Demonstração da avaliação de fatores externos identificados nos quintais caseiros na região de Machipanda.

\begin{tabular}{|c|c|c|c|c|}
\hline \multicolumn{2}{|c|}{$\begin{array}{c}\text { Fatores críticos de } \\
\text { sucesso }\end{array}$} & Peso & Desempenho & Resultado \\
\hline \multirow{4}{*}{ Oportunidades } & Fator 1 & W1 & D1 & W1 x D1 \\
\hline & Fator 2 & W2 & D2 & W2 x D2 \\
\hline & $\ldots \ldots \ldots$ & $\ldots \ldots$ & $\ldots \ldots$ & $\ldots \ldots$ \\
\hline & $\ldots \ldots \ldots$ & ....... & $\ldots$. & ....... \\
\hline \multirow{4}{*}{ Ameaças } & .......... & ....... & ..... & ....... \\
\hline & .......... & ...... & ....... & $\ldots \ldots$ \\
\hline & $\begin{array}{c}\text { Fator } \\
\mathrm{n}-1\end{array}$ & Wn-1 & Dn-1 & $\begin{array}{l}\text { Wn-1 x } \\
\text { Dn-1 }\end{array}$ \\
\hline & Fator $\mathrm{n}$ & Wn & Dn & Wn x Dn \\
\hline Total & & - & - & \\
\hline
\end{tabular}

Para peso igual a zero $(0,0)$ o fator foi considerado menos importante para os membros da comunidade local, enquanto para o fator cujo peso foi um $(1,0)$, este foi considerado muito importante na região de pesquisa. Em seguida, os fatores externos foram submetidos à avaliação de desempenho que indicava o grau de importância que os membros da comunidade atribuiam a um determinado fator. No entanto, para a avaliação de desempenho, considerou-se como respostas que eram fornecidas pelos membros da comunidade, valores variando de 1-4. Atribuiu-se valor 1 ao fator que foi considerado como resposta insuficiente, 2 ao fator que teve resposta média, 3 ao fator de resposta acima da média, e 4 ao fator que teve resposta muito superior na região de estudo. Para a obtenção do resultado final para os fatores externos, fez-se a multiplicação do peso atribuído para cada fator com o seu desempenho 
ao final fez-se o somatório total. Para a avaliação final dos fatores externos ou internos, considerou-se como valor de comparação o valor médio 2,0. A região do estudo poderia ser considerada como não atrativa para o desenvolvimento de sistemas agroflorestais se o valor total dos fatores externos ou internos fosse menor que $o$ valor médio $(<2,0)$; moderadamente atrativa $(2,0-3,0)$; atrativa $(3,0-4,0)$ e muito atrativa $(>4,0)$.

\section{Resultados e discussão}

Na região de Machipanda verificou-se que os quintais caseiros são, na sua maioria, de forma irregular e com área total variando de 400 a $5.000 \mathrm{~m}^{2}$, com média de $770,3 \mathrm{~m}^{2}$. Esse valor é relativamente maior, quanto comparada com o valor médio de $677 \mathrm{~m}^{2}$ encontrado por Maroyi (2013) nos quintais caseiros no distrito de Zvishavane, Zimbábue. No entanto, Reis (2011), no estudo de práticas agroflorestais tradicionais nas aldeias de Nhamombe, Chua e Chinhamazizi na localidade de Penhalonga (Manica), observou que 60\% dos agricultores entrevistados praticam os SAF's em áreas menores que 1 ha, mas muitos contavam com áreas maiores, sendo que $31 \%$ usavam áreas que variavam de 1 a 2 ha, e $4 \%$ possuíam áreas entre 2 e 5 ha.

As diferenças no tamanho das áreas observadas em Machipanda podem sofrer influência principalmente de fatores sociais, como observado por César (2009). Segundo este autor, o tamanho da área ocupada é consequência de fatores econômico. Os fundadores (líderes) conseguiram se estabelecer em grandes áreas, enquanto que indivíduos não nativos da região (geralmente recém-chegados à comunidade) conseguiram obter espaços pequenos devido à elevação do preço da terra.

Dentre os componentes agroflorestais encontrados nos quintais, verificou-se que o agrícola era o principal, estando presentes de forma isolada ou associada a outros componentes (Tabela 2).

Tabela 2. Atividades praticadas nos quintais caseiros de Machipanda, Moçambique.

\begin{tabular}{lcc}
\hline \multicolumn{1}{c}{ Práticas culturais } & $\begin{array}{c}\text { Número de } \\
\text { famílias }\end{array}$ & $\%$ \\
\hline Culturas agrícolas & 1 & 2,70 \\
Culturas agrícolas + animais & 6 & 16,2 \\
$\begin{array}{l}\text { SAFs (culturas agrícolas/ } \\
\text { fruteiras/animais) }\end{array}$ & 30 & 81,1 \\
\hline Total & 37 & 100 \\
\hline
\end{tabular}

Além da produção agrícola dos quintais caseiros, todas as famílias afirmaram possuir outras áreas longe das casas, denominadas machambas, geralmente destinadas à produção agrícola. As machambas na região de Machipanda apresentam boas condições de fertilidade e disponibilidade de água/umidade. As áreas são relativamente maiores que os quintais caseiros, variando de 0,5 ha a 2 ha, e são destinadas apenas à produção agrícola.

Nos quintais caseiros em Machipanda, constatouse que o milho era a cultura predominante em todos os quintais. Molebatsi et al. (2010) comparando a diversidade de plantas em quintais rurais e urbanos em Batswana (África do sul), constataram que das 70 espécies existentes nos quintais, de 20 a 30 eram indígenas e somente $19 \%$ constituíam culturas alimentares, sendo o milho (38\%) a cultura dominante e base da dieta das famílias. Isto mostra que os produtos obtidos nos diferentes quintais de comunidades da região austral da África apresentam o milho como a cultura mais frequente.

O componente animal foi dividido em animais de grande porte (bois) e de pequeno porte (cabritos, galinhas, perus, porcos, pombos, coelhos e/ou patos). A presença de animais foi observada em $97,3 \%$ dos quintais, sendo criados totalmente soltos, parcialmente soltos ou confinados.

Os animais mais comuns nos SAFs de Machipanda foram galinhas, cabritos e bois com 70\%, 54\% e 30\% respectivamente (Figura 1). Namwata et al. (2012), no seu estudo sobre a produtividade dos sistemas agroflorestais e sua contribuição na renda familiar dos agricultores no distrito de Lushoto, na Tanzânia, mostraram que as galinhas, vacas, caprinos, ovinos e patos foram os animais mais criados, com $88 \%$, $46,5 \%, 43,8 \%, 22,4 \%$ e $9,7 \%$, respectivamente. Esses autores revelaram ainda que a criação de galinhas era a preferida por ser relativamente fácil e barata de gerenciar, uma vez que não necessitava de capital inicial elevado quanto comparada com a criação dos outros animais.

O componente arbóreo encontrado nos quintais foi composto por 25 espécies, pertencentes a 17 famílias (Tabela 3). As famílias mais representativas foram: Rutaceae, Fabaceae, Anacardiaceae, Moraceae e Myrtaceae. As demais famílias contribuíram com apenas uma espécie cada. 


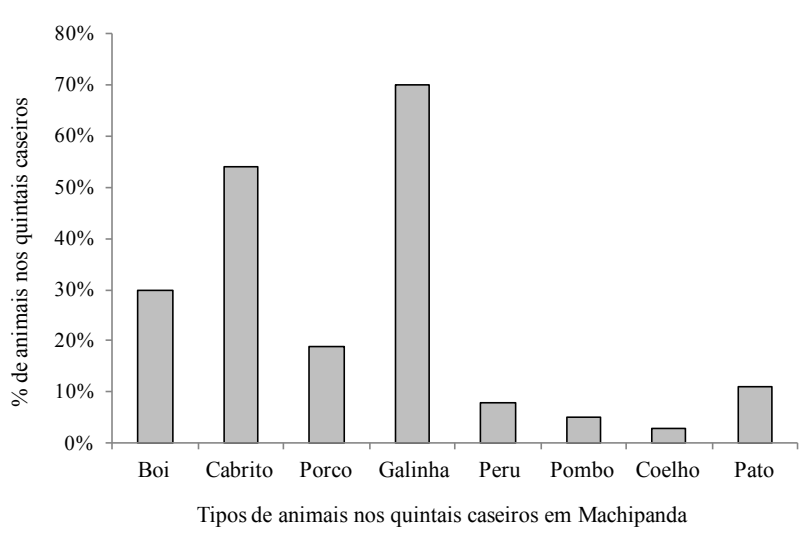

Figura 1. Distribuição de animais nos quintais caseiros de Machipanda, Moçambique.

Embora em muitos quintais tenha-se verificado indivíduos da mesma espécie, pode-se considerar os quintais caseiros de Machipanda pobres em diversidade de espécies arbóreas. Fato similar foi constatado por Maroyi (2013) nos quintais caseiros no distrito de Zvishavane, Zimbábue, onde observou a presença de 38 famílias botânicas com as seguintes famílias mais representativas: Poaceae (7 espécies), Fabaceae (6 espécies), Solanaceae (6 espécies), Brassicaceae (5 espécies), Cucurbitaceae (5 espécies) e Euphorbiaceae (3 espécies).

Das espécies arbóreas cultivadas nos quintais em Machipanda, verificou-se que a mangueira (Mangifera indica) e o abacateiro (Persea americana) foram as mais frequentes, sendo estas encontradas respectivamente em $62,2 \%$ e $43,4 \%$ dos quintais. Resultados similares foram verificados por Maroyi (2009) no estudo sobre quintais caseiros em Nhema, Zimbábue, que observou que a Mangifera indica, Persea americana e Citrus sinensis foram as árvores mais comuns na região do seu estudo. A frequência destas espécies nos quintais em Machipanda deve-se provavelmente ao relativo valor comercial de seus frutos nos mercados locais, além da facilidade de obtenção de variedades domesticadas que possibilitam início de produção de frutos precoce, além do cultivo dessas espécies ser uma prática cultural herdada.

Em Machipanda, as espécies arbóreas são utilizadas para sombreamento $(93 \%)$, ornamentação $(7 \%)$, alimentação (90\%), lenha (7\%), construção (7\%) e também para fins medicinais $(3 \%)$.
O uso de espécies frutíferas nos quintais caseiros em Machipanda contribui para a complementação alimentar das comunidades. Comportamento semelhante foi constatado em comunidades de outras regiões do mundo, como no Brasil, no estado do Mato Grosso do Sul (Amaral \& Guarim Neto, 2008), na Amazônia (Lourenço et al., 2009) e na Índia (Das \& Das, 2005).

A maioria dos chefes dos agregados familiares $(97,3 \%)$ relatou que em seus quintais não eram feitas práticas como desbaste e adubação química, sendo apenas efetuado o raleio e a capina quando as áreas continham culturas agrícolas. Fato também constatado por Santiago (2004), que ao estudar as características dos sistemas agroflorestais tradicionais das comunidades ribeirinhas do estado do Amazonas, no Brasil, verificou que os quintais recebiam poucos tratos culturais como capina, desbaste e podas ou adubação orgânica com resíduos da propriedade ou floresta. Esse autor observou também que quando estas atividades aconteciam, eram realizadas por mulheres e crianças.

\section{Análise dos fatores internos e externos identificados em Machipanda}

As forças e as fraquezas identificadas em Machipanda são fatores internos que ocorreram na região de estudo e que estavam sob o controle dos agricultores, enquanto que as oportunidades e as ameaças (fatores externos) são decorrentes do âmbito político e que fogem na maioria dos casos do controle ou ação direta dos agricultores (Tabelas 4 e 5). Para as características identificadas e agrupadas para as matrizes de fatores internos e externos obtiveram-se pesos ponderados totais de 2,61 e 3,01, respectivamente.

Pela análise de fatores internos comparados com os valores da classificação de fatores internos (Tabela 4) observa-se que a região de Machipanda possui quintais caseiros com moderada aptidão para a prática de SAFs. Isto significa é necessário aproveitar melhor as forças, de modo a reduzir as fraquezas.

A maioria da população rural moçambicana depende da produção agrícola para a complementação da dieta alimentar familiar e para obtenção de renda. A troca de experiência entre os membros da comunidade na produção de cultivos agrícolas, arbóreos, bem como na criação de animais, mostra a internalização social dessas práticas, com a manutenção dos costumes nas zonas rurais, potencializando a sua transferência para as gerações futuras. 
Tabela 3. Espécies encontradas nos quintais caseiros de Machipanda, Moçambique, percentual de agregados familiares e uso principal.

\begin{tabular}{|c|c|c|c|c|}
\hline Nome comum & Nome científico & Família & $\begin{array}{c}\text { \% do número } \\
\text { de agregados } \\
\text { familiares }\end{array}$ & Usos \\
\hline Abacate & Persea americana Mill. & Lauraceae & 43,2 & Fruto \\
\hline Acácia amarela & Senna seamea (Lamarck) Irwin \& Barneby & Fabaceae & 18,9 & Sombra \\
\hline Amora & Morus rubra $\mathrm{L}$. & Moraceae & 8,1 & Fruto \\
\hline Ata & Annona squamosa L. & Annonaceae & 2,7 & Fruto \\
\hline Banana & Musa acuminata Colla & Musaceae & 10,8 & Fruto \\
\hline Bauhinia & Bauhinia sp. & Fabaceae & 5,4 & Sombra \\
\hline Beijo de mulata & Catharanthus roseus L. & Apocinaceae & 2,7 & Medicinal \\
\hline Cajú & Anacardium occidentale L. & Anacardiaceae & 2,7 & Fruto e Sombra \\
\hline Casuarina & Casuarina equisetifolia $\mathrm{L}$. & Casuarinaceae & 2,7 & Estacas para construção \\
\hline Eucalipto & Eucalyptus sp. & Myrtaceae & 8,1 & Construção e lenha \\
\hline Goiaba & Psidium guajava $\mathrm{L}$. & Myrtaceae & 10,8 & Fruto \\
\hline Jacarandá & Jacaranda mimosifolia D. Don & Bignoniaceae & 8,1 & Ornamentacão \\
\hline Jatropha & Jatropha curcas L. & Euphorbiaceae & 2,7 & Sem uso \\
\hline Laranja & Citrus sinensis Osbeck & Rutaceae & 5,4 & Fruto e sombra \\
\hline Lichia & Litchi chinensis Sonn. Mill. & Sapindaceae & 8,1 & Fruto \\
\hline Limão & Citrus limon (L.) Burm & Rutaceae & 24,3 & Fruto \\
\hline Manga & Mangifera indica $\mathrm{L}$. & Anacardiaceae & 62,17 & Fruto \\
\hline Massala & Strychnus spinosa Lam. & Loganiaceae & 5,4 & Fruto \\
\hline Messassas & Brachystegia sp. & Fabaceae & 13,5 & Sombra \\
\hline Muzhanje & Casimiroa edulis & Rutaceae & 21,6 & Fruto \\
\hline Papaia & Carica papaya $\mathrm{L}$. & Caricaceae & 27,0 & Fruto \\
\hline Pêssego & Prunus persica L. Batsch & Rosaceae & 13,5 & Fruto \\
\hline Tangerineira & Citrus reticulata Blanco & Rutaceae & 5,4 & Fruto e sombra \\
\hline Tulia & Milicia excelsa (Welw.) C. Berg & Moraceae & 2,7 & Ornamentação \\
\hline Videira & Vitis vinifera $\mathrm{L}$. & Vitaceae & 13,5 & Fruto \\
\hline
\end{tabular}

A agregação cultural observada na região de estudo mostra que as comunidades ainda têm potencial para sustentar as suas famílias a partir dos produtos regionais. O uso de tecnologias melhoradas e a disponibilidade de assistência técnica são algumas medidas que poderiam contribuir para a melhoria da capacidade de produção. A disponibilização de assistência técnica de boa qualidade aos pequenos produtores possibilitaria o melhor aproveitamento e rentabilidade dos quintais e também das machambas.

A análise dos fatores externos mostra que a região de estudo apresenta maiores oportunidades $(2,48) \mathrm{em}$ relação às ameaças $(0,53)$. Isto demonstra que, apesar das dificuldades, como deficiências para a conservação da produção ou a falta de meios de transporte para escoamento dos produtos agrícolas para as cidades consumidoras, a região de Machipanda dispõe de características atrativas para o desenvolvimento de SAFs, como solos férteis, maior adaptabilidade das espécies arbóreas/frutíferas e maior área de criação de animais. Essas atividades podem ser potencializadas e exploradas de modo a contribuir para o desenvolvimento da comunidade local e do País.

O total dos valores ponderados obtidos para os fatores externos $(3,01)$ indica que a região de Machipanda apresenta fatores externos favoráveis à produção diversificada dos quintais e à oferta comercial de múltiplos produtos. 
Tabela 4. Matriz dos fatores internos sobre os quintais caseiros em Machipanda, Moçambique.

\begin{tabular}{|c|c|c|c|}
\hline Fatores internos & Peso & Desempenho & Peso ponderado \\
\hline \multicolumn{4}{|l|}{ Fortalezas } \\
\hline Garantia de segurança alimentar & 0,10 & 4 & 0,40 \\
\hline Aproveitamento de margens do rio para manutenção contínua da produção & 0,08 & 3 & 0,24 \\
\hline Troca de experiências entre os membros da comunidade & 0,06 & 4 & 0,24 \\
\hline Valorização cultural dos produtos produzidos nos quintais caseiros & 0,10 & 4 & 0,40 \\
\hline Disseminação e manutenção das práticas tradicionais & 0,06 & 4 & 0,24 \\
\hline Conhecimento da importância da integração da componente arbórea & 0,09 & 4 & 0,36 \\
\hline \multicolumn{4}{|l|}{ Fraquezas } \\
\hline Agricultura com tecnologias e práticas rudimentares & 0,06 & 2 & 0,12 \\
\hline Pouca variedade de espécies arbóreas nos quintais & 0,07 & 1 & 0,07 \\
\hline Perda de produtividade nos quintais ao longo do tempo & 0,08 & 1 & 0,08 \\
\hline Falta de condições para conservação da produção pós-colheita & 0,05 & 2 & 0,10 \\
\hline Baixo valor recebido pelos produtos produzidos nos quintais & 0,07 & 1 & 0,07 \\
\hline Falta de assistência técnica & 0,05 & 2 & 0,10 \\
\hline Falta de organização dos produtores & 0,06 & 2 & 0,12 \\
\hline Dificuldades na obtenção de sementes e mudas de espécies arbóreas & 0,07 & 1 & 0,07 \\
\hline Total & 1,00 & & 2,61 \\
\hline
\end{tabular}

Desempenho: 1 - Fraqueza maior; 2 - Fraqueza menor; 3 - Menor força; 4 - Maior força

Tabela 5. Matriz dos fatores externos sobre quintais caseiros em Machipanda, Moçambique.

\begin{tabular}{|c|c|c|c|}
\hline Fatores externos & Peso & Desempenho & Peso ponderado \\
\hline \multicolumn{4}{|l|}{ Oportunidades } \\
\hline Identidade cultural consolidada & 0,05 & 3 & 0,15 \\
\hline Garantia de conservação da biodiversidade local & 0,12 & 4 & 0,48 \\
\hline Tradição de produção agrícola e consumo dos produtos ofertados & 0,11 & 4 & 0,44 \\
\hline Potencialidade para produção orgânica & 0,09 & 4 & 0,36 \\
\hline Sustentabilidade do processo produtivo & 0,10 & 4 & 0,40 \\
\hline $\begin{array}{l}\text { Potencialidade para o desenvolvimento de associativismo e cooperativismo na } \\
\text { comunidade }\end{array}$ & 0,07 & 3 & 0,21 \\
\hline $\begin{array}{l}\text { Potencial para difusão e adotabilidade de SAFs como meio para o desenvolvimento rural } \\
\text { sustentável }\end{array}$ & 0,11 & 4 & 0,44 \\
\hline \multicolumn{4}{|l|}{ Ameaças } \\
\hline Dificuldade para escoamento da produção & 0,09 & 1 & 0,09 \\
\hline Deficiência no armazenamento de excedentes produtivos & 0,10 & 2 & 0,20 \\
\hline Êxodo rural na procura de melhores condições de vida & 0,08 & 2 & 0,16 \\
\hline $\begin{array}{l}\text { Disseminação de doenças em humanos devido à criação de animais em estruturas } \\
\text { inadequadas e próximas a casa principal }\end{array}$ & 0,08 & 1 & 0,08 \\
\hline Total & 1,00 & & 3,01 \\
\hline
\end{tabular}

Desempenho: 1 = Maior ameaça; 2 = Menor ameaça; 3 = Menor oportunidade; 4 = Maior oportunidade. 


\section{Conclusões}

As modalidades de SAFs praticadas nos quintais caseiros avaliados são de natureza agrossilvipastoril e silviagrícola.

A mangueira e o abacateiro são as espécies arbóreas mais frequentes nos quintais caseiros em Machipanda. Entre os animais, as galinhas, os bois e os cabritos são os preferidos.

Os quintais caseiros contribuem para a complementação alimentar dos agricultores e de suas famílias.

A troca de experiência entre os agricultores contribui para a disseminação de boas práticas de agricultura, assim como dos valores culturais dos agregados familiares.

A região de estudo mostra-se adequada para o desenvolvimento e intensificação dos quintais com mais pontos positivos que negativos, o que pode explicar a persistência destes sistemas ao longo do tempo.

A análise de fatores externos e internos dos quintais indica que a prática dos mesmos deve ser incentivada na região com base em princípios agroflorestais de sustentabilidade social, econômica e ambiental.

\section{Referências}

ABDO, M. T. V. N.; VALERI, S. V.; MARTINS, A. L. M. Sistemas agroflorestais e agricultura familiar: uma parceria interessante. Revista Tecnológica \& Inovação Agropecuária, p. 50-59, 2008.

ANTE, A. O. Agroforestería: aportes conceptuales, metodológicos y prácticos para el estúdio agroflorestal. Cali: Asociación del Colectivo de Agroecología del Suroccidente Colombiano, 2006. 238 p. (Serie Agroforestería).

AMARAL, C. N.; GUARIM NETO, G. Os quintais como espaços de conservação e cultivo de alimentos: um estudo na cidade de Rosário Oeste (Mato Grosso, Brasil). Ciências Humanas, Belém, v. 3, n. 3, p. 329-341, 2008.

CÉSAR, C. O. J. El huerto familiar como sistema agroforestal en Francisco villa, Tihuatlán, Veracruz. 2009. 170 f. Tesis (Inserir o título: Doutorado em...), Chapingo, México.

DAS, T.; DAS, A. K. Inventorying plant bio-diversity in homegardens: a case study in Barak Valley, Assam, North East India. Current Science, n. 89, p.155-163, 2005.

DUBOIS, J. Classificação e breve caracterização de SAFs e práticas agroflorestais. 1. In: MAY, P. H.; TROVATTO, C. M. M. Manual agroflorestal para a Mata Atlântica. Brasília, DF: Ministério do Desenvolvimento Agrário, 2008. p. 17-126.
FRAXE, T. J. P.; PEREIRA, H. S.; WITKOSKI, A. C. Comunidades ribeirinhas amazônicas: modos de vida e uso dos recursos naturais. Manaus: EDUA, 2007. 224 p.

FREITAS, C. G.; ROSA, L. S.; MACEDO, R. L. G. Características estruturais e funcionais dos quintais agroflorestais da comunidade Quilombola de Abacatal-Pará. In: CONGRESSO BRASILEIRO DE SISTEMAS AGROFLORESTAIS, 5., 2004, Curitiba. SAFs: desenvolvimento com proteção ambiental: anais. Colombo: Embrapa Florestas, 2004. p. 518-520. (Embrapa Florestas. Documentos, 98).

GAZEL FILHO, A. B.; YARED, J. A. G.; MOURÃO JUNIOR, M.; SILVA, M. F. da.; BRIENZA JUNIOR, S.; FERREIRA, G.; SILVA, P. de T. E. da. Diversidade e similaridade entre a vegetação de quintais agroflorestais em Mazagão, AP. In: CONGRESSO BRASILEIRO DE SISTEMAS AGROFLORESTAIS, 7., 2009, Luziánia. Diálogo e integração de saberes em sistemas agroflorestais para sociedades sustentáveis. [Luziânia]: Sociedade Brasileira de Sistemas Agroflorestais; [Brasília, DF]: EMATER-DF: Embrapa, 2009.

LOURENÇO, J. N. P.; SOUSA, S. G. A.; WANDELLI, E. V.; LOURENÇO, F. S.; GUIMARÃES, R. R.; CAMPOS, L. S.; SILVA, R. L.; MARTINS, V. F. C. Agrobiodiversidade nos Quintais Agroflorestais em Três Assentamentos na Amazônia Central. Revista Brasileira de Agroecologia, v. 4, n. 2, p. 965-969, 2009.

MACUCULE, A. Introdução a gestão participativa de recursos naturais. Maputo: IUCN Office, 2005. 91 p.

MAROYI, A. Traditional homegardens and rural livelihoods in Nhema, Zimbabwe: a sustainable agroforestry system. International Journal of Sustainable Development \& World Ecology, v. 16, n. 1, p. 1-8, 2009.

MAROYI, A. Use and management of homegard en plants in Zvishavane district, Zimbabwe. Tropical Ecology, v. 54, n. 2, p.191-203, 2013.

MOÇAMBIQUE. Ministério da Administração Estatal. Perfil do distrito de Manica, província de Manica. 2005. 60 p.

MOLEBATSI, L. Y.; SIEBERT, S. J.; CILLIERS, S. S.; LUBBE, C. S.; DAVOREN, E. The Tswana tshimo: a homegarden system of useful plants with a particular layout and function. African Journal of Agricultural Research, v. 5, n. 21, p. 2952-2963, 2010.

NAIR, P. K. R. An introduction to agroforestry. Dordrecht: Kluwer Academic Publishers, 1993. 497 p.

NAMWATA, B. M. L.; MASANYIWA, Z. S.; MZIRAI, O. B.; Productivity of the Agroforestry Systems and its contribution to Household Income among Farmers in Lushoto District, Tanzania. International Journal of Physical and Social Sciences, v. 2, p. 369-3924, 2012.

REIS, S. J. C. Práticas agroflorestais tradicionais acima de 1000 $\mathrm{m}$ de altitude nas aldeias de Nhamombe, Chua e Chinhamazizi na Localidade de Penhalonga. 2011. 68 f. Projeto de Licenciatura. Universidade Eduardo Mondlane, Maputo. 
SANTIAGO, J. L. Sistemas agroflorestais tradicionais e a sustentabilidade social das comunidades ribeirinhas do estado do Amazonas. In: V CONGRESSO BRASILEIRO DE SISTEMAS AGROFLORESTAIS, 5., 2004, Curitiba. SAFs: desenvolvimento com proteção ambiental: anais. Colombo: Embrapa Florestas, 2004. p. 96-101. (Embrapa Florestas. Documentos, 98).
TUZINE, M. S. Mapeamento da cobertura e uso da terra a partir de imagens ikonos na floresta de Inhamacari, província de Manica - Moçambique. 2011. 91 f. Dissertação (Mestrado em Ciências Florestais) - Universidade Federal do Paraná, Curitiba.

VAN LEEUWEN, J. Possibilidades e dificuldades para a agrossilvicultura na Amazônia, reflexões à base de experiências brasileiras. In: PAIVA, R.; BERTHA, L. (Org.). Lineas estrategicas y áreas temáticas en investigación agroforestal en la amazonía: Seminario Agroforestería para el desarrollo ambiental de la región. Florência-Caquetá: Universidad de la Amazonia, 2007. 6 p. 
